\title{
Homozygous Missense Variation in PNPLA8 Causes Prenatal-Onset Severe Neurodegeneration
}

\author{
Suzena Masih Amita Moirangthem Shubha R. Phadke \\ Department of Medical Genetics, Sanjay Gandhi Post Graduate Institute of Medical Sciences, Lucknow, India
}

\section{Established Facts}

- PNPLA8 is associated with mitochondrial myopathy and poor weight gain with lactic acidosis.

- Previously reported patients had truncating variations that lie outside the conserved patatin domain of PNPLA8.

\section{Novel Insights}

- The authors report a patient with prenatal-onset severe and progressive neurodegeneration with variation in PNPLA8.

- The patient presented in this report harbors a novel missense variant in the patatin domain of PNPLA8.

\section{Keywords}

Congenital microcephaly · Neurodegenerative disorder .

Patatin domain - Whole-exome sequencing - PNPLA8

\section{Abstract}

The patatin-like protein family plays an important role in various biological functions including lipid homeostasis, cellular growth, and signaling. Conserved across species, the patatin domain is shared by all 9 members of the PNPLA family without redundancy in the coding sequences. The defective function of PNPLA2, PNPLA6, and PNPLA9 are known to cause mitochondrial-related neurodegeneration. Recently, PNPLA8 has been associated with mitochondrial myopathy and poor weight gain with lactic acidosis in 3 unrelated families. Using whole-exome sequencing, we identified a homozygous novel missense variation c.1874A $>\mathrm{G}$ in the patatin domain of PNPLA8. The patient had prenatal-onset severe and progressive neurodegeneration with mortality in infancy.

(c) 2021 S. Karger AG, Base

\section{Introduction}

Mitochondriopathies are a group of clinically heterogeneous genetic disorders caused either by sporadic or inherited variants in genes positioned on nuclear or mitochondrial DNA. The majority of proteins involved in mitochondrial function are encoded by nuclear genes. Decreased mitochondrial respiration is seen in cases with defective 


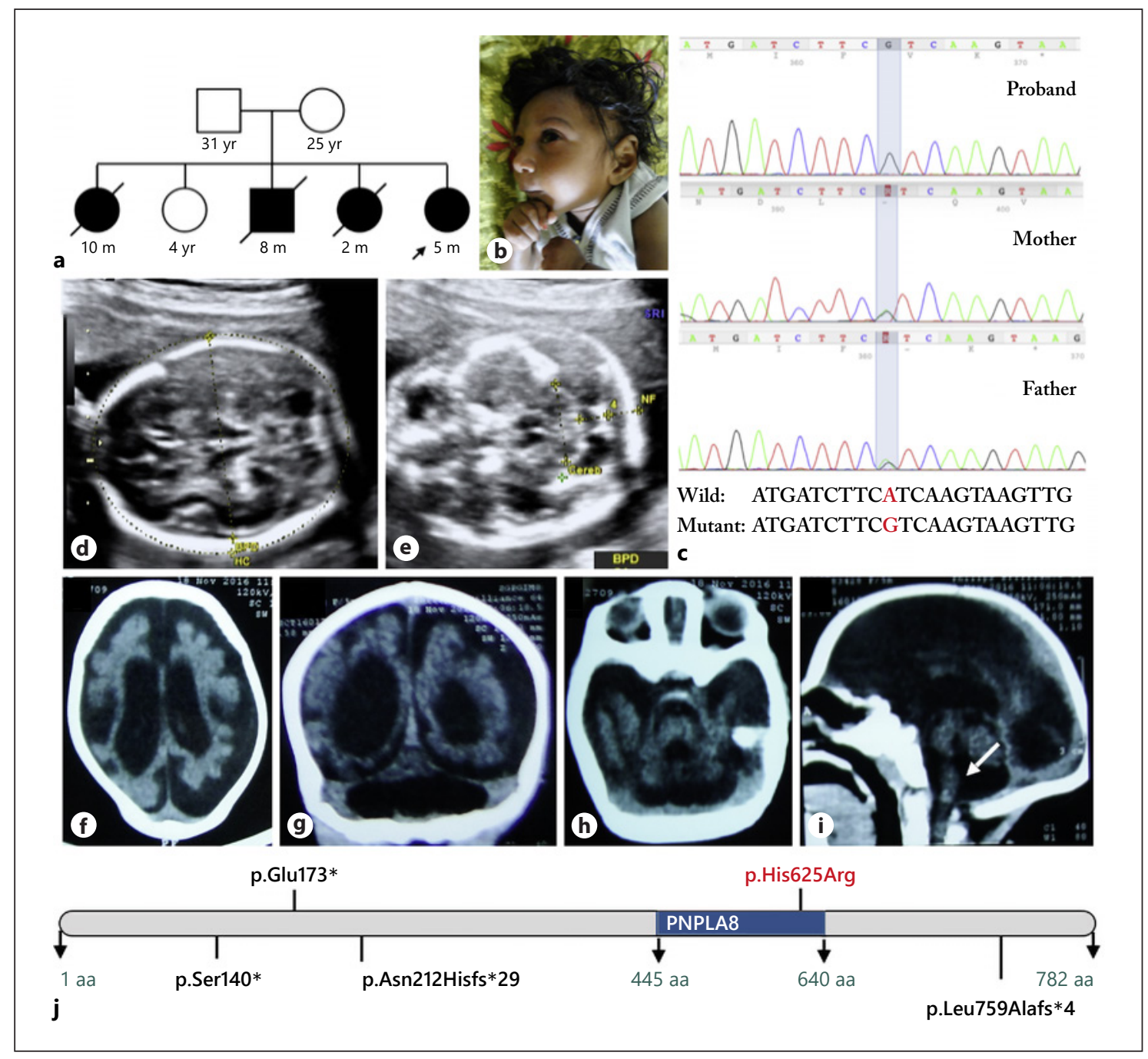

Fig. 1. a Pedigree of the family (black arrow indicates the proband). b Proband at 5 months of age with microcephaly, receding forehead, thin upper lip, low-set ears and neck hyperextension. c Sequence chromatogram of the proband showing homozygous c.1874A > G in PNPLA8 (NM_015723) and carrier parents. Ultrasonography done at gestational age of 20 weeks showed $\mathbf{d}$ normal

phospholipases A2, which is essential for normal mitochondrial function. PNPLA8 is one of the predominant calcium-independent phospholipases in mammalian mitochondria. Variations in this gene have been associated with mitochondrial myopathy and poor weight gain with lactic acidosis (OMIM 251950) [Saunders et al., 2015; Shukla et al., 2018]. Here, we report a fourth patient with a sequence variation in PNPLA8 with prenatal-onset microcephaly, intractable seizures, and spasticity. Our patient harbored a homozygous missense variation in contrast to previous patients who had nonsense sequence variations.

PNPLA8-associated Prenatal-Onset

Severe Neurodegeneration cerebral development and e cerebellar hypoplasia. Postnatal CT scan of the proband at 5 months of age showed atrophy of cerebral hemispheres (f) and cerebellum ( $\mathbf{g}-\mathbf{i})$. Hypoplasia of pons is also noted (white arrow in i). $\mathbf{j}$ Schematic representation of PNPLA8 with the previously reported variants (black) and in our patient (red).

\section{Case Presentation}

A nonconsanguineous married woman presented at 20 weeks 1 day of pregnancy with a history of the death of 3 offspring during infancy (shown in Fig. 1a). All of them had intractable seizures with onset during the neonatal period. They did not achieve any developmental milestones. During this pregnancy, ultrasonography at 20 weeks showed normal cerebral development (Fig. 1d) and cerebellar hypoplasia (Fig. 1e). At 21 week 3 days, ultrasonographic pictures of the fetal brain showed cerebellar atrophy (transverse cerebellar diameter of $-3.2 \mathrm{Z}$ score) and head circumference $(16.7 \mathrm{~cm})$ less than $-2 Z$ score. The microcephaly and cerebellar hypoplasia continued to progress during fetal life. At the 
gestational age of 24 weeks, the fetus' head circumference was $19.14 \mathrm{~cm}(-3 Z$ score $)$ and abdominal circumference was $18.65 \mathrm{~cm}$ $(-0.7 \mathrm{Z}$ score). A female baby was delivered at term having a birth weight of $2.5 \mathrm{~kg}$ ( $-1.7 \mathrm{Z}$ score). Record of head circumference at birth was not available. She was in a neonatal intensive care unit soon after birth with intractable seizures and generalized hypertonia. She was brought to us at 5 months of age for an evaluation. She had severe global developmental delay and had not achieved any milestones. She had a receding forehead, thin upper lip, low-set ears, and neck hyperextension (Fig. 1b). Her length was $55 \mathrm{~cm}(-4$ $Z$ score), weight was $3 \mathrm{~kg}$ ( $-5 Z$ score), and her head circumference was $32 \mathrm{~cm}$ (-8 $Z$ score). She had generalized spasticity and opisthotonic posturing. CT of the brain showed a shriveled brain (Fig. 1f) and atrophy of the cerebellar hemispheres (Fig. 1g-i). Comparison with images of prenatal ultrasonography suggested a progressive loss of brain matter and architecture. The child died at 6 months of age. Phenotype and neuroimaging were suggestive of pontocerebellar hypoplasia, especially type 4 and 5 (caused by variations in TSEN54), which are known to have onset during prenatal life [Patel et al., 2006; Namavar et al., 2011].

\section{Materials and Methods}

Genomic DNA was extracted from peripheral blood lymphocytes using QIAamp DNA Extraction Mini Kit. Chromosomal microarray was done using Affymetrix CytoScan $750 \mathrm{~K}$ array (Affymetrix, Inc. Santa Clara, CA, USA). Whole-exome sequencing (WES) of the proband was performed on Illumina NextSeq500 platform after library preparation and capture using Illumina Nextera Rapid Capture Exome Kit V1.2 (Illumina, Inc., San Diego, CA, USA). Bioinformatics analysis was done using an in-house pipeline based on BWA-mem, Picard tools, GATK, and Annovar. The coverage of genes known to be causative of pontocerebellar hypoplasia phenotype was also examined. Sanger sequencing of the variant identified was performed on ABI 3100Dx Genetic Analyzer (Applied Biosystems, Foster City, CA, USA).

\section{Results}

\section{Chromosomal Microarray}

No pathogenic/likely pathogenic copy number loss/ gain was identified in our patient. Taking $3 \mathrm{Mb}$ as laboratory cut-off value, a total region of $72 \mathrm{Mb}$ with loss of heterozygosity was identified. However, this region did not have any genes relevant to the phenotype observed in our patient.

\section{Whole-Exome Sequencing}

Prioritizing for biallelic variants based on family history, a homozygous missense variation c.1874A $>\mathrm{G}$ (p. His625Arg) in PNPLA8 (NM_015723) was identified in the proband. The variant has not been reported in mutation databases like ClinVar and HGMD. This variation has a minor allele frequency of 0.00003184 ( 1 heterozygous carrier of the European non-Finnish population) in gnom $A D$. It is not present in 1000 genomes and in our in-house exomes of 727 individuals. This variation was predicted as deleterious by various in silico prediction tools (MutationTaster, Provean, SIFT, and PolyPhen2). Sanger sequencing of exon 7 was done to validate the results of WES in the proband and showed both parents to be heterozygous for the variant (Fig. 1c). No other variation relevant to the patient's phenotype was detected in the WES data.

\section{Discussion}

We report a patient with a novel missense sequence variation in PNPLA8 characterized by severe and progressive neurodegeneration with documented prenatal onset. The patient had documented microcephaly and cerebellar hypoplasia on second trimester ultrasonography. Postnatal CT scan showed shriveled cerebellum and cerebrum. Recently, Shukla et al. [2018] reported 2 unrelated individuals with biallelic variations in PNPLA8. Microcephaly was reported prenatally in one patient with neonatal-onset multifocal clonic seizures but it was more severe in our patient (Table 1). The other patient with compound heterozygous variation achieved all milestones until 13 months after which she developed seizures, neuroregression and progressive weakness, and involuntary movement. She shared clinical similarity with the first patient reported by Saunders et al. [2015], who developed progressive mitochondrial myopathy with dystonia at 1 year of age.

PNPLA8 is a calcium-independent phospholipases A2 (iPLA2s) belonging to a patatin-like protein family and shares homology with patatin, a lipid hydrolase. Having mitochondrial and peroxisomal localization signals in the $\mathrm{N}$-terminal and C-terminal, respectively, it is the predominant phospholipase active in the mammalian mitochondria and peroxisome and is thought to have a role in maintaining lipid homeostasis in these organelles [Hara et al., 2019]. Presently, 9 PNPLA genes (PNPLA1PNPLA9) are known in humans [Kienesberger et al., 2009]. While most members of PNPLA family (PNPLA2, PNPLA3, PNPLA5, and PNPLA7) have their role in lipid storage and metabolism [Fischer et al., 2007; Reilich et al., 2011; Mikhailova et al., 2019; Pingitore and Romeo, 2019; Wang et al., 2020], PNPLA1 is associated with congenital ichthyosis (OMIM \#612121). Labonne et al. [2020] recently proposed PNPLA4 as likely genes for X-linked in- 
Table 1. Clinical and molecular findings in patients with pathogenic variation in PNPLA8

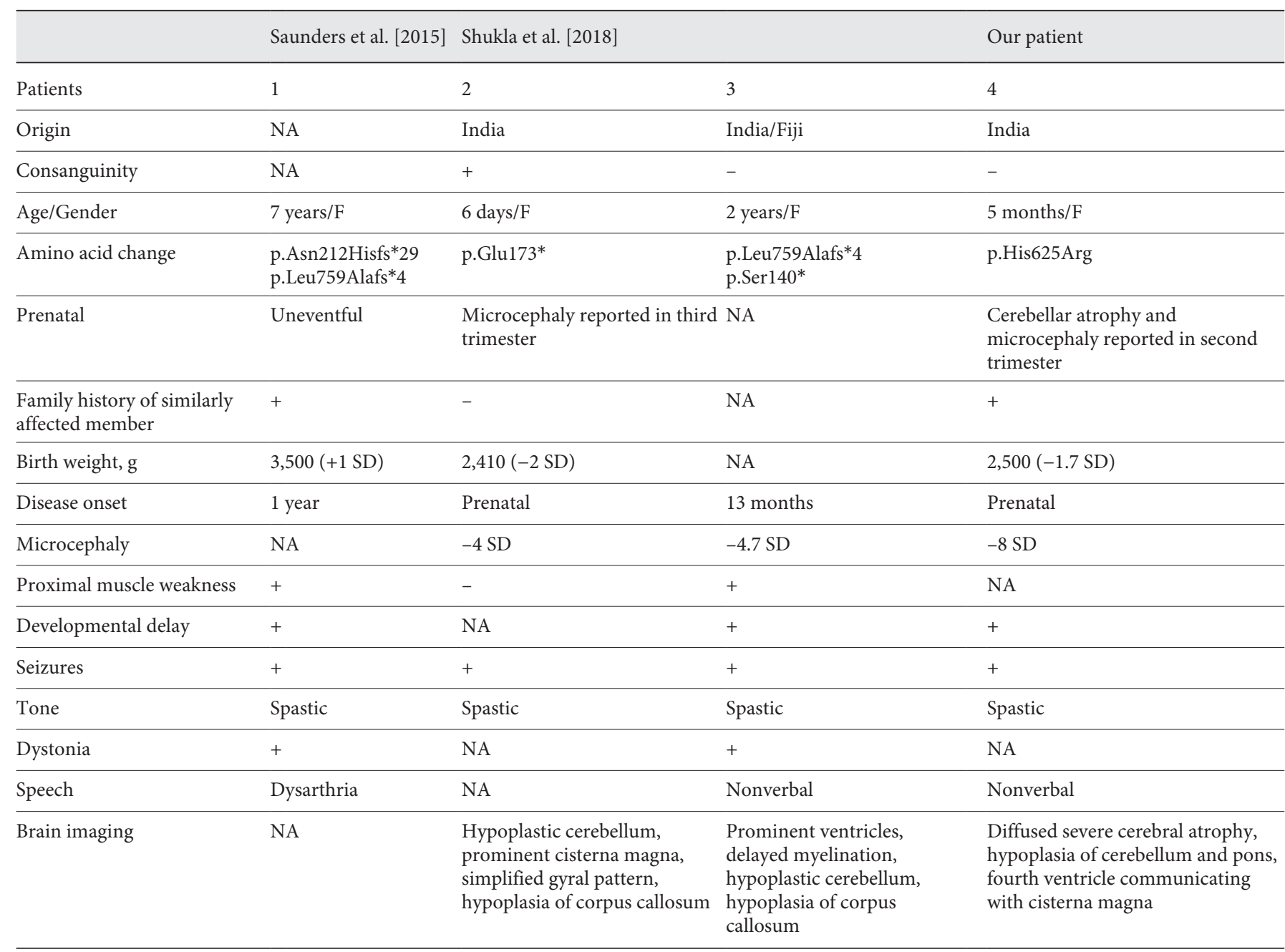

NA, not available; SD, standard deviation; + present, - absent.

tellectual disability based on comparative genomic mapping study. Variations in PNPLA6 are reported to cause Oliver-McFarlane syndrome (OMIM\# 275400), Laurence-Moon syndrome (OMIM\# 245800), and BoucherNeuhauser syndrome (OMIM\# 215470). These disorders have variable overlapping features of intellectual impairment, hypogonadism, and retinal degeneration. Additionally, PNPLA6 variations are also causative of spastic paraplegia type 39 (OMIM\# 612020). Variations in PNPLA9 consequently result in neurodegeneration with brain iron accumulation 2B (OMIM\# 610217), infantile neuroaxonal dystrophy 1 (OMIM\# 256600), and Parkinson disease type 14 (OMIM\# 612953).
Our patient harbored a missense variant in the patatin domain of PNPLA8. All 3 previously reported patients had truncating variations that lie outside this domain (Fig. 1j). The patatin domain is shared by all 9 members of the PNPLA family without redundancy in the coding sequences and is conserved across species. Thus, it may be suggested that missense variation in the patatin domain can result in severe phenotypes and early infantile lethality comparable to truncating variations outside it (Table 1). A similar phenotype was recapitulated in mouse models with PNPLA8 null variants [Mancuso et al., 2009]. The cellular consequence of missense variation in the patatin domain, however, remains to be elucidated by functional validation. 


\section{Acknowledgement}

The authors thank the family for participating in this study.

\section{Statement of Ethics}

The study was approved by the institutional ethics committee. Informed written consent was obtained from the parents for genetic testing and publication of clinical details and photographs.

\section{Conflict of Interest Statement}

The authors have no conflicts of interest to declare.

\section{Funding Sources}

This study was supported by the Indian Council of Medical Research (Grant No. 63/8/2010-BMS).

\section{Author Contributions}

Suzena Masih drafted the manuscript and performed the Sanger sequencing of the variant identified in WES. Whole-exome data analysis and variant identification was done by Amita Moirangthem. Clinical evaluation of the presented case and overall supervision of the study was done by Shubha R Phadke. All authors discussed the results and contributed to the final manuscript.

\section{References}

Fischer J, Lefèvre C, Morava E, Mussini JM, Laforêt $\mathrm{P}$, Negre-Salvayre A, et al. The gene encoding adipose triglyceride lipase (PNPLA2) is mutated in neutral lipid storage disease with myopathy. Nat Genet. 2007;39(1):28-30.

Hara S, Yoda E, Sasaki Y, Nakatani Y, Kuwata H. Calcium-independent phospholipase A2 $\gamma$ (iPLA2 $\gamma$ ) and its roles in cellular functions and diseases. Biochim Biophys Acta Mol Cell Biol Lipids. 2019;1864(6):861-8.

Kienesberger PC, Oberer M, Lass A, Zechner R. Mammalian patatin domain containing proteins: A family with diverse lipolytic activities involved in multiple biological functions. J Lipid Res. 2009;50(Suppl 1):S63-8.

Labonne JDJ, Driessen TM, Harris ME, Kong I-K, Brakta S, Theisen J, et al. Comparative Genomic Mapping Implicates LRRK2 for Intellectual Disability and Autism at 12q12, and HDHD1, as Well as PNPLA4, for X-Linked Intellectual Disability at Xp22.31. J Clin Med. 2020;9(1):274.
Mancuso DJ, Kotzbauer P, Wozniak DF, Sims HF, Jenkins CM, Guan S, et al. Genetic ablation of calcium-independent phospholipase A2 \{gamma\} leads to alterations in hippocampal cardiolipin content and molecular species distribution, mitochondrial degeneration, autophagy, and cognitive dysfunction. J Biol Chem. 2009;284(51):35632-44.

Mikhailova S, Ivanoshchuk D, Timoshchenko O, Shakhtshneider E. Genes Potentially Associated with Familial Hypercholesterolemia. Biomolecules. 2019;9(12):807.

Namavar Y, Chitayat D, Barth PG, van Ruissen F, de Wissel MB, Poll-The BT, et al. TSEN54 mutations cause pontocerebellar hypoplasia type 5. Eur J Hum Genet. 2011;19(6):724-6.

Patel MS, Becker LE, Toi A, Armstrong DL, Chitayat D. Severe, fetal-onset form of olivopontocerebellar hypoplasia in three sibs: $\mathrm{PCH}$ type 5?. Am J Med Genet A. 2006;140(6):594603.

Pingitore P, Romeo S. The role of PNPLA3 in health and disease. Biochim Biophys Acta Mol Cell Biol Lipids. 2019;1864(6):900-6.
Reilich P, Horvath R, Krause S, Schramm N, Turnbull DM, Trenell M, et al. The phenotypic spectrum of neutral lipid storage myopathy due to mutations in the PNPLA2 gene. J Neurol. 2011;258(11):1987-97.

Saunders CJ, Moon SH, Liu X, Thiffault I, Coffman K, LePichon JB, et al. Loss of function variants in human PNPLA8 encoding calcium-independent phospholipase A2 $\gamma$ recapitulate the mitochondriopathy of the homologous null mouse. Hum Mutat. 2015;36(3): 301-6.

Shukla A, Saneto RP, Hebbar M, Mirzaa G, Girisha KM. A neurodegenerative mitochondrial disease phenotype due to biallelic loss-offunction variants in PNPLA8 encoding calcium-independent phospholipase A2 $\gamma$. Am J Med Genet A. 2018;176(5):1232-7.

Wang X, Gou M, Wang Q, Qingjie W, Zou S, et al. The patatin-like phospholipase domain containing protein 7 facilitates VLDL secretion by modulating ApoE stability. Hepatology. 2020;72(5):1569-85. 